\title{
How to Deal with Measures of Association: A Short Guide for the Clinician
}

\author{
M.J. Knol ${ }^{a} \quad$ A. Algraa, $\quad$ R.H.H. Groenwold ${ }^{a}$ \\ a Julius Center for Health Sciences and Primary Care, University Medical Center Utrecht, and ${ }^{b}$ Department of \\ Neurology and Neurosurgery, Rudolf Magnus Institute of Neuroscience, Utrecht Stroke Center, University Medical \\ Center Utrecht, Utrecht, The Netherlands
}

\section{Key Words}

Risk ratio $\cdot$ Rate ratio $\cdot$ Number needed to treat $\cdot$ Hazard ratio $\cdot$ Odds ratio

\begin{abstract}
When reading medical literature as a clinician, many different measures of association are presented. To judge whether results of studies can be applied to clinical practice, it is essential to understand and to be able to interpret the measure of association reported in the article. In this paper, we will present how to deal with the most commonly used measures of association including the risk and rate difference, number needed to treat, risk and rate ratio, hazard ratio and odds ratio. By means of examples, we will discuss the different measures of association for the three main study designs used in clinical research: randomized controlled trial, observational cohort study and case-control study.
\end{abstract}

Copyright $\odot 2011$ S. Karger AG, Basel

Suppose you read in the abstract of a recently published randomized controlled trial (RCT) that the odds ratio for the relation between treatment and survival was
3.5 with a $95 \%$ confidence interval of $1.5-8.2$. How would you interpret this odds ratio? That the risk of survival is 3.5 times higher with treatment than without? Or that the odds of survival is 3.5 times higher with treatment than without? But what is an odds? And how do you explain the results to your colleagues and patients? Actually, is the odds ratio the correct measure of association in an RCT?

When reading medical literature, you will find many different measures of association, the odds ratio being just one of them. To judge whether results of studies can be applied to clinical practice, it is essential to understand and to be able to interpret the measure of association reported in the article.

In this paper, we will present how to deal with the most commonly used measures of association including the risk and rate difference (both $\mathrm{RD}$ ), number needed to treat (NNT), risk and rate ratio (both RR), hazard ratio (HR) and odds ratio (OR). We will use the full names instead of the abbreviations in this paper for clarity. We will discuss the different measures of association for the three main study designs used in clinical research. Although we focus on effects of interventions, all remarks equally apply to effects of etiologic factors.

\section{KARGER \\ Fax +4161306 1234 E-Mail karger@karger.ch} www.karger.com
(C) 2011 S. Karger AG, Basel $1015-9770 / 12 / 0332-0098 \$ 38.00 / 0$

Accessible online at: www.karger.com/ced
M.J. Knol, $\mathrm{PhD}$

UMC Utrecht, Julius Center

PO Box 85500

NL-3508 GA Utrecht (The Netherlands)

Tel. +31 88755 1168, E-Mail m.j.knol@umcutrecht.nl 
Table 1. Patient numbers and outcomes in the three main study designs

a Number of patients with and without the primary outcome, and person-years of observation in both treatment groups in the RCT example [1]

\begin{tabular}{lllll}
\hline & \multicolumn{2}{l}{ Primary outcome } & \multirow{2}{*}{ Person-years } \\
\cline { 2 - 4 } & yes & no & total & \\
\hline Aspirin + dipyridamole & 173 & 1,190 & 1,363 & 4,498 \\
Aspirin alone & 216 & 1,160 & 1,376 & 4,495 \\
\hline
\end{tabular}

b Number of patients with and without a poor outcome, and person-years of observation in the intravenous thrombolysis group and antithrombotic treatment alone group in the observational cohort study example [2]

\begin{tabular}{lllll}
\hline & \multicolumn{2}{l}{ Poor outcome } & \multirow{2}{*}{ Person-years } \\
\cline { 2 - 4 } & yes & no & total & \\
\hline Intravenous thrombolysis & 71 & 50 & 121 & 10.08 \\
Antithrombotic treatment alone & 117 & 66 & 183 & 15.25 \\
\hline
\end{tabular}

c Number of subjects with and without current oral contraceptive use in 203 stroke patients and 916 control women in the case-control study example [3]

\begin{tabular}{lll}
\hline Current oral contraceptive use & \multicolumn{2}{l}{ Stroke } \\
\cline { 2 - 3 } & yes & no \\
\hline Yes & 102 & 348 \\
No & 101 & 568 \\
\hline Total & 203 & 916 \\
\hline
\end{tabular}

\section{Clinical Examples}

There are three main study designs used in clinical research to investigate the effects of medical interventions: RCT, observational cohort study and case-control study. Below, we discuss each of these and the measures of association that can be calculated in these designs.

\section{Randomized Controlled Trial}

The ESPRIT study group [1] studied the effect of aspirin plus dipyridamole versus aspirin alone after cerebral ischemia of arterial origin. In this RCT, patients were assigned to aspirin (30-325 mg daily) with $(\mathrm{n}=1,363)$ or without $(\mathrm{n}=1,376)$ dipyridamole $(200 \mathrm{mg}$ twice daily) within 6 months of a transient ischemic attack or minor ischemic stroke of presumed arterial origin. The primary outcome was the composite of death from all vascular causes, nonfatal stroke, nonfatal myocardial infarction, or major bleeding complication, whichever happened first. The primary analysis was by intention to treat and the mean follow-up time was 3.2 years. Table 1a presents the number of patients with and without the primary outcome, and person-years of observation in both treatment groups. Person-years are the total amount of time that subjects are observed and at risk of getting the outcome, which is either until the outcome occurs, until loss to follow-up or until the end of the study. One person-year could mean that 1 person is followed for 1 year, or that 2 persons are followed for half a year.

\section{Observational Cohort Study}

Schonewille et al. [2] conducted an observational cohort study among 592 consecutive patients who presented with an acute symptomatic and radiologically confirmed basilar artery occlusion. Patients were divided into three groups according to the treatment they received: antithrombotic treatment only, which comprised antiplatelet drugs or systemic anticoagulation; primary intravenous thrombolysis, including subsequent intraarterial thrombolysis, or intra-arterial therapy, which 
comprised thrombolysis, mechanical thrombectomy, stenting or a combination of these approaches. Outcome was assessed at 1 month after acute basilar artery occlusion, and a poor outcome was defined as a modified Rankin scale score of 4 or 5 , or death. Table $1 b$ presents the number of patients with and without the primary outcome, and person-years of observation in the antithrombotic treatment and intravenous thrombolysis treatment groups (for simplicity we discard the data of the third treatment group).

\section{Case-Control Study}

Kemmeren et al. [3] studied the risk of ischemic stroke in relation to oral contraceptives. This was a populationbased case-control study including women aged 18-49 years. A total of 203 women with a first ischemic stroke were compared with 925 control women without vascular diseases. The control subjects were recruited by randomdigit dialing. All patients and control subjects filled in a questionnaire about the use of oral contraceptives and risk factors for ischemic stroke. Table 1c presents the number of subjects with and without current oral contraceptive use in 203 stroke patients and 916 control women (oral contraceptive use was unknown for 7 control women, and 2 control women used hormone replacement therapy).

\section{Measures of Association}

We will explain for the most commonly used measures of association how they can be calculated, how they should be interpreted, why they differ from the other measures (if applicable), and for which of the three study designs they can be calculated. For the observational studies (cohort and case-control design) we will only give unadjusted measures (i.e. not taking confounding into account), which allows us to make a direct link between the observed risks or rates and the measures of association. Table $2 \mathrm{a}-\mathrm{c}$ presents the calculations and estimates of the measures of association for the three example studies.

\section{Absolute Measures of Association}

\section{Risk Difference}

The risk difference is the risk of the outcome among treated subjects minus the risk of the outcome among untreated subjects and is typically expressed as a percentage. For the cohort study example, this is 71/121 - 117/183= $-0.053=-5.3 \%$ (table $2 \mathrm{~b}$ ). One could also say that the re- duction of the risk in an absolute sense (absolute risk reduction) is $5.3 \%$, and hence the risk difference reflects the absolute difference in risk of the outcome in the two treatment groups. The risk difference can be calculated in an RCT and cohort study but generally not in a case-control study. In a case-control study, the ratio between the cases and controls is determined by the investigator; thus, the proportion of cases in the study does not reflect the actual risk of the disease in the population. Only if the sampling fraction of the controls is known, the risk difference can be calculated in a case-control study. The same holds for the other measures we discuss, except for the odds ratio (see below). In the example case-control study, the sampling fraction was not known and therefore we could not calculate the risk difference in this particular casecontrol study.

\section{Rate Difference}

The rate difference is the number of treated patients with the outcome divided by the person-years of observation in that group (the rate) minus the number of untreated patients with the outcome divided by the person-years of observation in that group. Person-years are the total amount of time that subjects are observed and at risk of getting the outcome, which is either until the outcome occurs, until loss to follow-up or until the end of the study. In the RCT example, the rate difference was $173 / 4,498-216 / 4,495=-0.0096$ per person-year $=-0.96 \%$ per year (table 2a). Note that the unit of the rate difference is per year, whereas the risk difference was dimensionless. Especially when the follow-up time is not the same for all patients, or when there is loss to follow-up, the rate difference better reflects the effect of treatment than the risk difference. In our cohort study example, the follow-up time was the same for all patients, namely 1 month, and there was no loss to follow-up in this short time period. Therefore, the person-years are exactly the number of patients times 1 month of follow-up (table 1b), leading to a rate difference of $-63 \%$ per year or $-5.3 \%$ per month (table $2 b$ ), the latter being exactly the same as the risk difference. Note that if the rate difference is presented per year, it is assumed that extrapolation from 1 month to 1 year is appropriate.

Number Needed to Treat

The number needed to treat is the inverse of the risk difference or rate difference. It reflects how many patients need to be treated with the drug to prevent 1 outcome. In the RCT example, the number needed to treat based on the risk difference was $1 /(173 / 1,363-216 / 1,376)=34$ (ta- 
Table 2. Calculations and estimates of the measures of association

a For the RCT example [1]

\begin{tabular}{lll}
\hline Measure of association & Calculation & Estimate \\
\hline Absolute & & \\
$\quad$ Risk difference & $173 / 1,363-216 / 1,376$ & $-0.030=-3.0 \%^{\mathrm{a}}$ \\
$\quad$ Number needed to treat & $1 /(173 / 1,363-216 / 1,376)$ & 34 \\
$\quad$ Rate difference & $173 / 4,498-216 / 4,495$ & -0.0096 per person-year $=-0.96 \%$ per year \\
$\quad$ Number needed to treat & $1 /(173 / 4,498-216 / 4,495)$ & 105 per year \\
Relative & $(173 / 1,363) /(216 / 1,376)$ & $0.81^{\mathrm{b}}$ \\
$\quad$ Risk ratio & $(173 / 4,498) /(216 / 4,495)$ & 0.80 \\
$\quad$ Rate ratio & - & 0.80 \\
$\quad$ Hazard ratio & $(173 / 1,190) /(216 / 1,160)$ & $0.78^{\mathrm{c}}$ \\
$\quad$ Odds ratio &
\end{tabular}

b For the observational cohort study example [2]

\begin{tabular}{lll}
\hline Measure of association & Calculation & Estimate \\
\hline $\begin{array}{l}\text { Absolute } \\
\text { Risk difference }\end{array}$ & $71 / 121-117 / 183$ & \\
$\quad$ Number needed to treat & $1 /(71 / 121-117 / 183)$ & $-0.053=-5.3 \%^{\mathrm{d}}$ \\
$\quad$ & $71 / 10.08-117 / 15.25$ & -0.63 per person-year $=-63 \%$ per year $=$ \\
Rate difference & & $-5.3 \%$ per month \\
$\quad$ Number needed to treat & $1 /(71 / 10.08-117 / 15.25)$ & 2 per year; 19 per month \\
Relative & $(71 / 121) /(117 / 183)$ & $0.92^{\mathrm{e}}$ \\
Risk ratio & $(71 / 10.08) /(117 / 15.25)$ & 0.92 \\
$\quad$ Rate ratio & - & - \\
Hazard ratio & $(71 / 50) /(117 / 66)$ & $0.80^{\mathrm{c}}$ \\
Odds ratio & &
\end{tabular}

c For the case-control study example [3]

\begin{tabular}{lll}
\hline Measure of association & Calculation & Estimate \\
\hline Absolute & - & - \\
Risk difference & - & - \\
Number needed to treat & - & - \\
Rate difference & - & - \\
Number needed to treat & - & - \\
Relative & - & - \\
Risk ratio & - & - \\
Rate ratio & - & - \\
Hazard ratio & - & - \\
Odds ratio & $(102 / 101) /(348 / 568)$ & 1.65 \\
\hline
\end{tabular}

a Absolute risk reduction $=3.0 \%$.

${ }^{\mathrm{b}}$ Relative risk reduction $=(1-0.81) \times 100=19 \%$.

${ }^{\mathrm{c}}$ Not appropriate to calculate in RCTs or observational cohort studies [6].

$\mathrm{d}$ Absolute risk reduction $=5.3 \%$.

${ }^{\mathrm{e}}$ Relative risk reduction $=(1-0.92) \times 100=8 \%$. 
ble $2 a)$, meaning that 34 patients need to be treated with aspirin and dipyramidole to prevent 1 patient with a poor composite outcome within 3.2 years (the mean follow-up time). In contrast, the number needed to treat based on the rate difference was $1 /(173 / 4,498-216 / 4,495)=105$ per year (table 2a), meaning that 105 patients need to be treated with aspirin and dipyramidole to prevent 1 patient with a poor composite outcome within 1 year. As became apparent from this example, it is essential to know the time period to which the number needed to treat refers. Also note that a number needed to treat of 10 per 3 years is the same as a number needed to treat of 30 per year.

\section{Relative Measures of Association}

\section{Risk Ratio}

The risk ratio is the risk of the outcome among treated subjects divided by the risk of the outcome among untreated subjects. The risk ratio in the RCT example is $(173 / 1,363) /(216 / 1,376)=0.81$ (table 2a). This means that the risk of a poor outcome in the group treated with aspirin and dipyramidole is 0.81 times the risk of a poor outcome in the group treated with aspirin alone, which actually means that the group treated with combination therapy is better off. One could also say that the relative risk reduction is $(1-0.81) \times 100=19 \%$. The risk ratio is sometimes referred to as the relative risk.

\section{Rate Ratio}

The rate ratio is the number of treated patients with the outcome divided by the person-years of observation in that group divided by the number of untreated patients with the outcome divided by the person-years of observation in that group. For the RCT example, the rate ratio was $(173 / 4,498) /(216 / 4,495)=0.80$ (table $2 \mathrm{a})$, which can be interpreted similarly as a risk ratio: the rate of a poor outcome in the group treated with aspirin and dipyramidole is 0.80 times the rate of a poor outcome in the group treated with aspirin alone, i.e. a relative rate reduction of $20 \%$. Again, as for the rate difference compared with the risk difference, the rate ratio better reflects the effect of treatment than the risk ratio when follow-up time varies between patients. In our cohort study example, the followup time was exactly the same for all patients and therefore the risk ratio and rate ratio are exactly the same.

\section{Hazard Ratio}

The hazard ratio is essentially the same as the rate ratio. However, an assumption underlying the estimation of the rate ratio is that the rate is constant over time. If it is unlikely that this assumption holds, e.g. because the risk of complications is highest directly after surgery and reduced over time, one could apply a method that allows for a change in the hazard over time as long as the ratio of the hazards over time is constant. This latter condition is reflected in the name of the Cox proportional hazards model that is used to calculate the hazard ratio, as the hazard ratio cannot be calculated directly from the crude numbers. In the RCT example, the hazard ratio was 0.80 (table 2a), virtually the same as the rate ratio. The interpretation of the hazard ratio is also similar to the rate ratio: the hazard of a poor outcome in the group treated with aspirin and dipyramidole is 0.80 times the hazard in the group treated with aspirin alone, a relative reduction of $20 \%$.

\section{Odds Ratio}

The odds ratio is the odds of treatment (i.e. number of subjects with treatment divided by number of subjects without treatment) in the cases divided by the odds of treatment in the controls. In the case-control study example, the odds ratio is $(102 / 101) /(348 / 568)=1.65$ (table $2 \mathrm{c}$ ). Although the odds ratio is usually the only measure of association that can be calculated in a case-control study, it can be interpreted as a risk ratio or rate ratio, provided a certain sampling method of the controls is employed [4]. For example, if each time a case is diagnosed a control is selected from the population at risk at that point in time (also called incidence density sampling), the estimated odds ratio can be interpreted as a rate ratio. Irrespective of the sampling method, the odds ratio is a good approximation of the risk ratio if the outcome is rare, which is usually true for case-control studies.

In an RCT or cohort study, the odds ratio can be calculated as well. The odds ratio is then defined as the odds of the outcome in the treated patients divided by the odds of the outcome in the untreated patients. In an RCT or cohort study, however, the odds ratio only approximates the risk ratio if the outcome is rare or if the odds ratio is close to 1 . In the RCT example, the odds ratio is $(173 / 1,190) /$ $(216 / 1,160)=0.78$ (table 2a), whereas the risk ratio is 0.81 . The difference is small because in this RCT the outcome was relatively rare (14\%) and the odds ratio was close to one. In the cohort study example, the difference between the odds ratio and risk ratio was larger (odds ratio $=0.80$ vs. risk ratio $=0.92$; table $2 \mathrm{~b}$ ) because the outcome was not rare (44\%). Preferably, odds ratios should not be reported in RCTs and cohort studies to prevent any possible misinterpretation. If odds ratios are reported in an RCT or cohort study, be cautious to not interpret it as a risk ratio; especially if the outcome is not rare or the odds ratio is far from $1[5,6]$. 


\section{Conclusion}

In this paper, we have explained how to calculate and interpret the risk and rate difference, number needed to treat, risk and rate ratio, odds ratio and hazard ratio in different study designs. We hope this will help clinicians when reading medical literature. Regarding the questions we posed at the beginning of this article: the odds ratio of
3.5 can only be interpreted as 'the risk of survival is 3.5 times higher with treatment than without' if the outcome is rare. The risk or rate ratio and the risk or rate difference are the preferred measures of association in an RCT, not the odds ratio. Preferably the absolute risk or rate under treatment and under no treatment should be communicated to patients.

\section{References}

$>1$ The ESPRIT Study Group: Aspirin plus dipyridamole versus aspirin alone after cerebral ischaemia of arterial origin (ESPRIT): randomised controlled trial. Lancet 2006; 367:1665-1673.

2 Schonewille WJ, Wijman CA, Michel P, et al: Treatment and outcomes of acute basilar artery occlusion in the Basilar Artery International Cooperation Study (BASICS): a prospective registry study. Lancet Neurol 2009; 8:724-730.
-3 Kemmeren JM, Tanis BC, van den Bosch MA, et al: Risk of Arterial Thrombosis in Relation to Oral Contraceptives (RATIO) study: oral contraceptives and the risk of ischemic stroke. Stroke 2002;33:1202-1208.

4 Knol MJ, Vandenbroucke JP, Scott P, Egger M: What do case-control studies estimate? Survey of methods and assumptions in published case-control research. Am J Epidemiol 2008;168:1073-1081.
5 Knol MJ, Duijnhoven RG, Grobbee DE, Moons KG, Groenwold RH: Potential misinterpretation of treatment effects due to use of odds ratios and logistic regression in randomized controlled trials. PLoS One 2011; 6:e21248.

6 Knol MJ, Le Cessie S, Algra A, Vandenbroucke JP, Groenwold RH: Tackling the problem of overestimating risk ratios by odds ratios in randomized controlled trials and observational cohort studies: alternatives for logistic regression. CMAJ 2011, in press. 